\title{
Experience with Oseltamivir in the Control of Nursing Home Influenza A Outbreak
}

\author{
Noriharu Shijubo, Gen Yamada, Mamoru Takahashi, Tetsuya TokunoH*, Takashi Suzuki* and Shosaku Abe
}

\begin{abstract}
Objective Influenza outbreaks have revealed that elderly persons are a great risk of death and serious complications after infection. The administration of oseltamivir, a neuramidase inhibitor, is effective for prophylaxis of influenza and to reduce disease duration and severity in healthy adults with naturally acquired febrile influenza. To clarify the usefulness of oseltamivir in the elderly we administered oseltamivir to all residents when an influenza $A$ outbreak occurred in a nursing home.

Patients Sixty-eight residents in the nursing home were investigated in which the influenza A outbreak occurred; 32 residents had fever and 28 residents were positive for influenza A with direct enzyme immunoassay.

Methods Oseltamivir was administered at $75 \mathrm{mg}$ twice daily for $\mathbf{5}$ days to all residents.

Results Oseltamivir almost inhibited symptom onset in the influenza A-positive afebrile group. Initiation at 0 hour (22 cases), 1-12 hours (4 cases), 13-24 hours (5 cases) or 72 hours ( 1 case) from onset of symptoms was associated with mean fever durations of $26 \pm 18$ hours, $38 \pm 21$ hours, $54 \pm 12$ hours and 120 hours, respectively, indicating that earlier initiation of therapy was associated with faster resolution of fever in elderly patients. Oseltamivir may be effective for household prophylaxis in the elderly persons. Oseltamivir administration was well tolerated in elderly persons.
\end{abstract}

Conclusion Oseltamivir is effective for the reduction or prophylaxis of influenza $A$ infection in elderly persons. (Internal Medicine 41: 366-370, 2002)

Key words: elderly, neuraminidase inhibitor, prophylaxis

\section{Introduction}

Despite the development of antibiotics and vaccines, infec- tious diseases are a continuing threat to humans, especially the elderly. The age-associated decline of immune function contributes to the increased susceptibility of the aged to the infection (1). Influenza infection continues to inflict an important burden on health-care systems, filling hospital beds during winter months and causing misery to millions of people worldwide. Although vaccination can lessen the impact of the disease in nursing homes, efficacy can be variable and unvaccinated populations remain vulnerable to disease $(2,3)$.

Antivirals represent a rational approach to influenza control; now M2 inhibitors, (amantadine and rimantadine) and influenza neuramidase inhibitors (zanamivir and oseltamivir) are available. The use of the M2 inhibitors is limited by the potential for rapid emergence of resistant viruses capable of transmission to and causing disease in close contacts. Neither treatment is effective against influenza B, and amantadine has an adverse-effect profile that limits its use in frail elderly patients (4). Influenza neuramidase is essential for viral replication. Through cleaving of terminal sialic-acid residues from glycoproteins, this enzyme facilitates the release of new virus particles from infected cells, prevents virus aggregations, and promotes viral passage through respiratory mucus (5). Inhibition of the enzyme has become possible with the development of influenza neuramidase inhibitors zanamivir (6) and oseltamivir (7). Zanamivir has poor oral bioavailability and has been developed for administration by inhalation (8), a route that requires specific instructions to ensure adequacy of delivery. The oral prodrug, oseltamivir is highly bioavailable and undergoes rapid conversion to the active from after gastrointestinal absorption (9). Oseltamivir prophylaxis has been shown to be effective in protecting home residents from influenza and in halting the outbreak of influenza in the nursing home (10).

We experienced an influenza A outbreak in a nursing home; 32 cases out of 68 residents had fever and influenza A virus was positive in 28 residents by direct enzyme immunoassay of throat swab specimens. Outbreaks of influenza infection have shown that elderly persons are at a great risk of death and serious complications after influenza infection $(11,12)$. Here, in the nursing home only 1 resident had had a vaccination this

From *the Third Department of Internal Medicine, Sapporo Medical University School of Medicine, Sapporo and *the First Department of Internal Medicine Hokkaido Prefectural Esashi Hospital, Esashi

Received for publication October 30, 2001; Accepted for publication January 16, 2002

Reprint requests should be addressed to Dr. Noriharu Shijubo, the Third Department of Internal Medicine, Sapporo Medical University School of Medicine, South-1, West-16, Chuo-ku, Sapporo 060-8543 
winter season. The oral prodrug oseltamivir $(75 \mathrm{mg}$ twice daily for 5 days) was administrated to 68 residents for reduction of influenza A infection or for prophylaxis of influenza A infection. We analyzed the efficacy and safety of the agent to treat acute influenza in the elderly patients.

\section{Patients and Methods}

\section{Influenza $A$ outbreak in the nursing home}

There were 68 residents ( 18 men and 50 women, mean age, 81 years with range of 64 to 96 years) in the nursing home. Out of 68 residents 9 residents had heart diseases, 8 lung diseases, and 24 cerebral vascular diseases. Out of 68 residents, 28 were bedridden. The nursing home had 23 staff ( 4 men and 19 women, mean age, 41 years with range of 23 to 58 years) for care of the residents. Only 1 resident had had the influenza A vaccination the previous November. Because of high-grade fever, 5 residents visited an outpatient clinic of respiratory medicine of Hokkaido prefectural Esashi hospital in the morning of April 12, 2001. Four out of the 5 residents were positive for influenza $A$ in direct enzyme immunoassay (Directigen FluA, Becton Dickinson Co., Sparks, MD, USA) of throat swab specimens. We decided to examine all residents in the nursing home in the outpatient clinic that afternoon. We took medical histories, measured vital signs, and did physical examinations. Thirteen residents had a high-grade fever $\left(38^{\circ} \mathrm{C} \leq\right)$ and 19 , a low-grade fever $\left(37^{\circ} \mathrm{C} \leq, 38^{\circ} \mathrm{C}>\right.$, Fig. 1). Time distribution after symptom onset showed 0 hour in 22 cases, 1-12 hours in 4 cases, 13-24 hours in 5 cases and 72 hours in 1 case. Zero hour

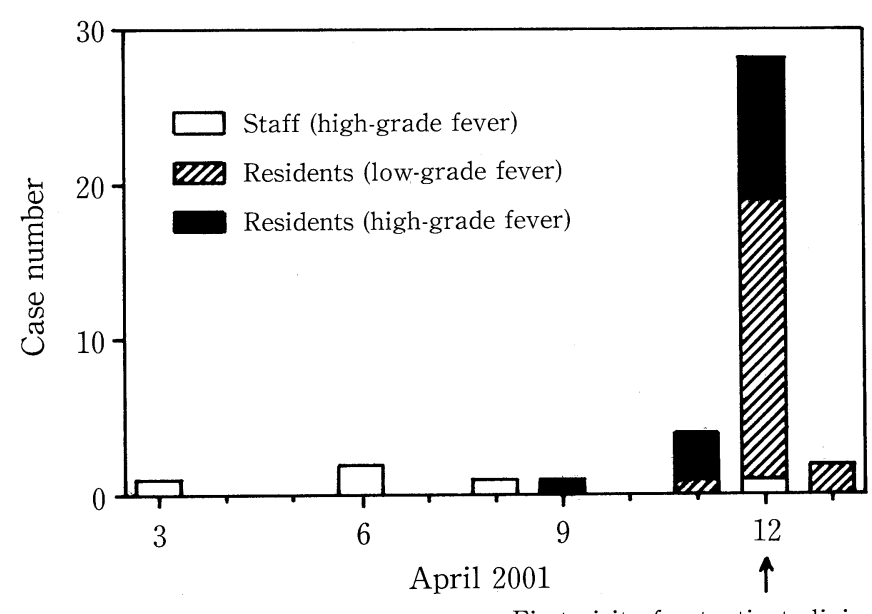

First visit of outpatient clinic

Figure 1. Outbreak of influenza in a nursing home. There were 68 residents in the nursing home. Thirteen residents had a highgrade fever $\left(38^{\circ} \mathrm{C} \leq\right)$ and 19 , a low-grade fever $\left(37^{\circ} \mathrm{C} \leq, 38^{\circ} \mathrm{C}>\right)$ from April 9 to 12, 2001. Ten residents had a fever before their visit to the outpatient clinic and 22 residents were noted to have a fever at the time of outpatient visit. Four staff had a high-grade fever before the outbreak of influenza $A$ infection. On April 13, 2 residents who had no fever at the time of outpatient visit showed low-grade fever. means that a resident did not notice an elevation of body temperature until the outpatient clinic. Four staff had a high-grade fever before the outbreak of influenza A infection.

\section{Examination of the residents and oseltamivir therapy for all residents}

Chest X-ray films, common blood cell counts, biochemistry, C-reactive protein (CRP) and influenza A virus of throat swab specimens by direct enzyme immunoassay were tested in all the residents. Oral oseltamivir (75 $\mathrm{mg}$ twice daily) was administrated for 5 days in all the residents for reduction or for household prophylaxis of influenza A infection. Body temperature was measured at least twice a day in all residents. Five days after the therapy, chest X-ray films, common blood cell counts, biochemistry, and CRP were also tested in all the residents. Common blood cell counts, biochemistry and CRP were frequently tested in the residents with high-grade fever within 28 days after the therapy.

Informed content was obtained from patients or their family.

\section{Statistical analysis}

Data were expressed as mean \pm SD. The paired Student's $t$ test was used to compare paired sets of data. $p<0.05$ was considered to be statistically significant.

\section{Results}

\section{Influenza A virus detection by direct enzyme immunoassay of throat swab specimens}

Influenza A virus was positive in 28 of 68 residents by direct enzyme immunoassay of throat swab specimens. According to the magnitude of fever, 10 of 13 residents with highgrade fever were positive for influenza A virus and 8 of 19 residents with low-grade fever were positive for influenza $A$ virus (Fig. 2). Ten residents without fever $\left(37^{\circ} \mathrm{C}>\right)$ were positive for influenza $\mathrm{A}$ virus and 14 residents with fever were negative for influenza A virus in direct enzyme immunoassay of throat swab specimens. We divided the residents into four groups: influenza A-positive febrile (18 cases), influenza Anegative febrile (14 cases), influenza A-positive afebrile (10 cases) and influenza A-negative afebrile groups ( 26 cases). We speculated that influenza A affected most residents in the influenza A-negative febrile group since the direct immunoassay of throat swab specimens gave some false negative results. One resident (influenza A positive) had pneumonia and severe dehydration was observed in two residents ( 1 case positive and 1 case negative for influenza A virus); they were admitted. Oral oseltamivir $75 \mathrm{mg}$ twice daily was administrated for 5 days in 3 admitted cases and 65 residents. The patient with pneumonia was administered intravenous antibiotics.

\section{Efficacy of oseltamivir therapy}

The efficacy of oseltamivir therapy was evaluated to monitor body temperature at least twice a day because few residents complained of respiratory symptoms such as sore throat $(5$ 


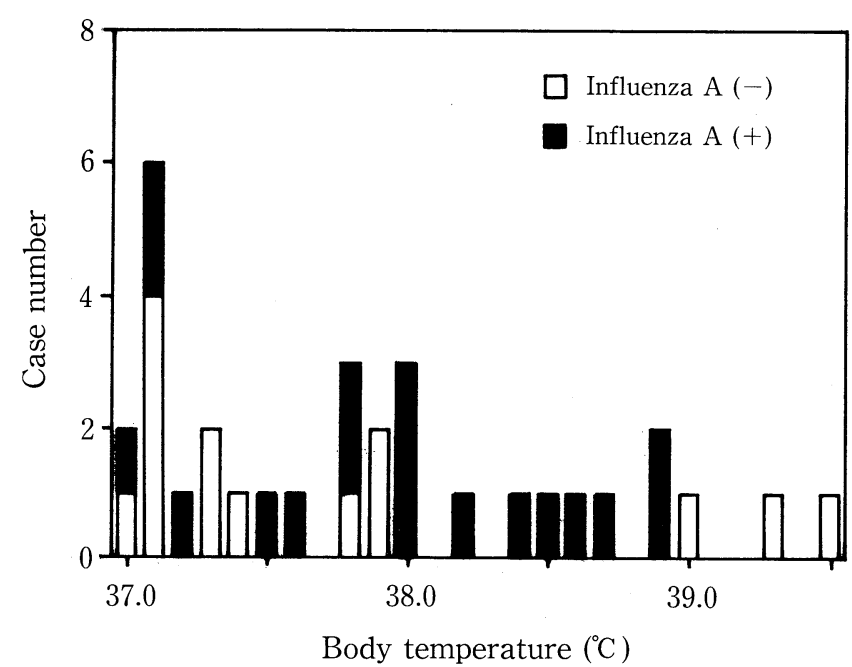

Figure 2. Distribution of body temperature in the influenza Apositive and -negative febrile groups. Influenza $A$ virus was positive in 28 of 68 residents by direct immunoassay of throat swab specimens. We divided into four groups: influenza A-positive febrile (18 cases), influenza $A$-negative febrile (14 cases), influenza $A$-positive afebrile (10 cases) and influenza $A$-negative afebrile groups (26 cases). Influenza $A$ was positive in 10 of 13 residents with high-grade fever $\left(38^{\circ} \mathrm{C} \leq\right)$ and 8 of 19 residents with lowgrade fever $\left(37^{\circ} \mathrm{C} \leq, 38^{\circ} \mathrm{C}>\right)$.

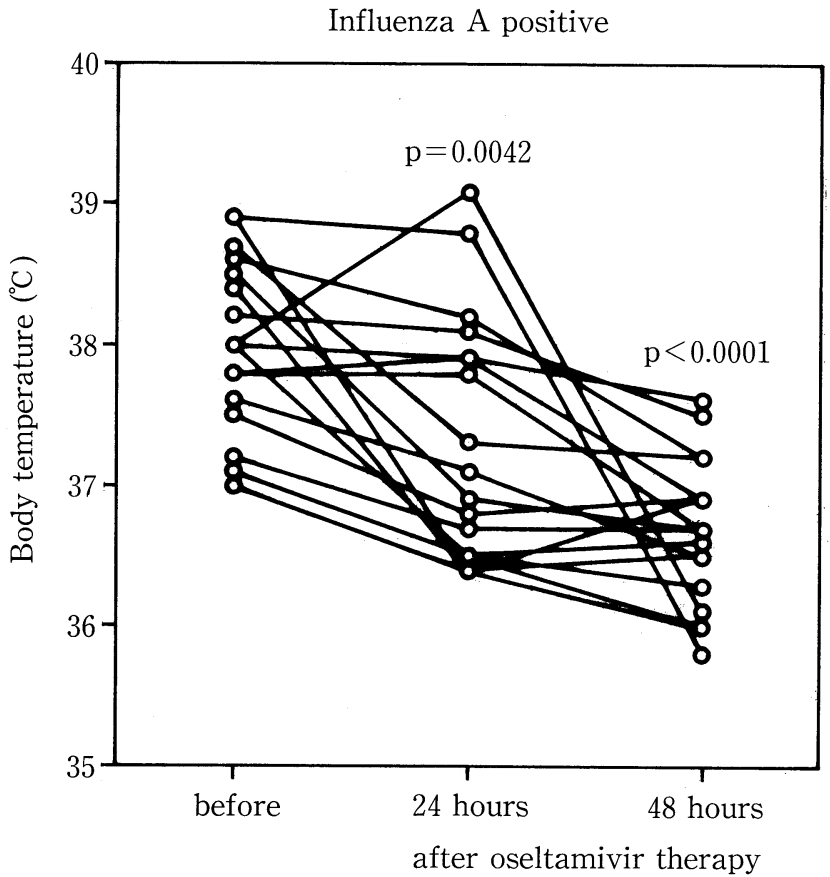

cases), cough ( 3 cases) and nasal symptoms ( 2 cases). Figure 3 depicts body temperature before and 24 hours and 48 hours after oseltamivir therapy in influenza A-positive and -negative febrile groups. In the influenza A-positive febrile group, after oseltamivir administration, 50\% (9 cases) became afebrile (went down below $37^{\circ} \mathrm{C}$ ) within 24 hours and $78 \%$ (14 cases) did so within 48 hours. In the influenza A-negative group 64\% (9 cases) became afebrile within 24 hours and all of them did so within 48 hours after therapy. In 13 cases of high-grade fever, $46 \%$ (6 cases) became afebrile within 24 hours and $69 \%$ ( 9 cases) did so within 48 hours after therapy. The remaining three cases recovered from their illness within 72 hours and one case treated 72 hours after symptom onset needed 120 hours to become afebrile. In 19 cases of low-grade fever, $63 \%$ (12 cases) became afebrile within 24 hours and $94 \%$ ( 18 cases) recovered within 48 hours after therapy. The remaining one case became afebrile 72 hours after the therapy. The patient with pneumonia became afebrile 72 hours after therapy and two admitted patients with dehydration also recovered 72 hours after the therapy. Initiation at 0 hour ( 22 cases), $1-12$ hours ( 4 cases), 13-24 hours (5 cases) or 72 hours ( 1 case) from onset of symptoms was associated with mean fever durations of $26 \pm 18$ hours, $38 \pm 21$ hours, $54 \pm 12$ hours and 120 hours, respectively. In the influenza A-positive afebrile group, 2 residents had a low-grade fever $\left(37.2^{\circ} \mathrm{C}\right.$ and $\left.37.4^{\circ} \mathrm{C}\right) 24$ hours after the therapy, but these residents became afebrile 36 hours after the therapy. The remaining 34 afebrile residents ( 8 positive for influenza $A$ and 26 negative for influenza $A$ ) had neither fever nor influenza-

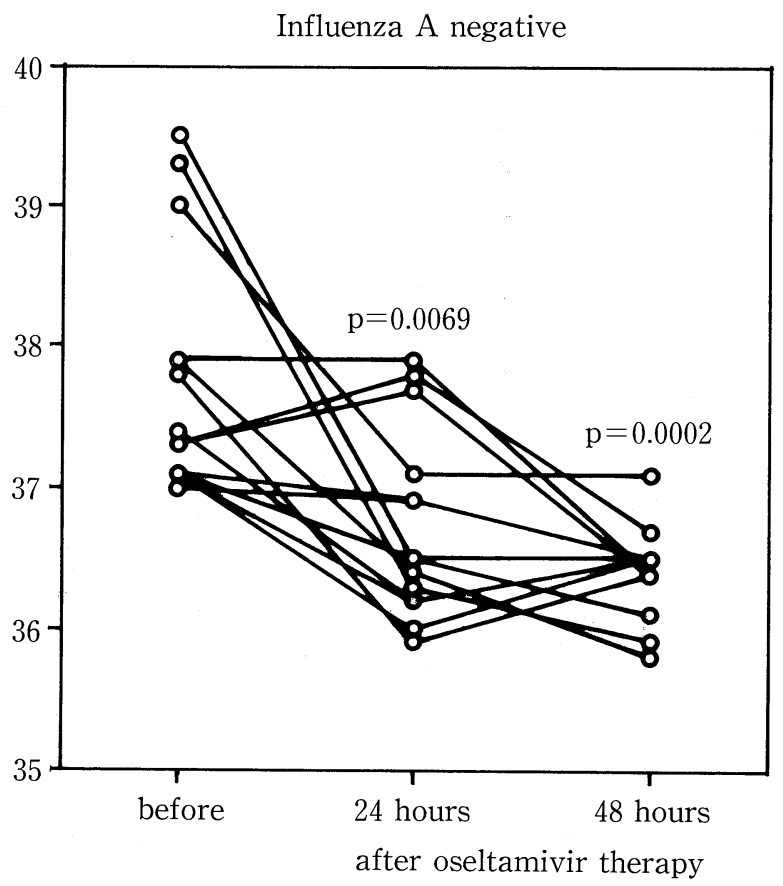

Figure 3. Body temperature before and 24 hours and 48 hours after oseltamivir therapy in influenza A-positive and -negative febrile groups. Body temperature 24 hours and 48 hours after oseltamivir therapy was significantly reduced as compared with that before the therapy in influenza A-positive and -negative febrile groups. The paired Student's t test was used to compare paired sets of data. 
like symptoms during the therapy (for 120 hours). All residents were followed 28 days after initiation of oseltamivir therapy, none of them had fever or influenza-like symptoms in this period.

\section{Tolerability}

None of them showed significant adverse events including upper gastrointestinal events during the courses of oseltamivir therapy, when oseltamivir was taken with food. No significant abnormal changes of laboratory findings 5 days after initiation of oseltamivir therapy were found as compared with those before oseltamivir therapy.

\section{Discussion}

Aging is a complex process that affects a wide variety of body functions including those of the immune system (13). Decreased delayed-type hypersensitivity response, lymphocyte proliferation, and cytotoxic $\mathrm{T}$ lymphocyte activity and increased memory $\mathrm{T}$ lymphocytes, and antibody response plus altered cytokine patterns are among the changes observed with aging (14). Influenza virus is one of the most infectious agents in humans and outbreaks of influenza showed that elderly persons are at a great risk of death and serious complications after influenza infection $(11,12)$.

Oseltamivir is a prodrug of oseltamivir carboxylate, a potent and selective inhibitor of the neuramidase glycoprotein essential for replication of influenza A and B viruses (9). Studies in volunteers with experimental human influenza A or B showed that administration of oral oseltamivir 20 to $200 \mathrm{mg}$ twice daily for 5 days reduced both the quantity and duration of viral shedding compared with placebo (15). Subsequent assessment of the drug at a dosage of $75 \mathrm{mg}$ twice daily for 5 days in otherwise healthy adults with naturally acquired febrile influenza showed that oseltamivir reduced the duration of the disease by up to 1.5 days and the severity of illness by up to $38 \%$ compared with placebo when initiated within 36 hours of symptom onset (16-18). Oseltamivir prophylaxis was reported to be very effective in protecting from influenza like illness and in halting the outbreak of influenza $B$ in the nursing home (residents' mean age 78 years) (10).

We described an outbreak of influenza $A$ in the nursing home (68 residents); 13 residents showed high-grade fever (10 influenza A-positive) and 19 residents low-grade fever (8 influenza A-positive) and influenza $A$ was positive in 10 afebrile residents in the first visit of outpatient clinic. Only 1 resident had had the influenza A vaccination that winter. Vaccination can lessen the impact of the disease in the elderly persons $(19,20)$, but most of the residents had not taken vaccination in this winter season. We could examine most cases within 24 hours from symptom onset, and administrated oseltamivir $75 \mathrm{mg}$ twice daily for 5 days in all residents for reduction of infection or for household prophylaxis of influenza A infection. We demonstrated that oseltamivir administration was associated with significant clinical improvement in the elderly persons with naturally occurring influenza $A$ (18 cases). Oseltamivir therapy was also effective for febrile elderly patients in whom influenza A was negative in throat swab tests by direct enzyme immunoassay. We speculated that influenza A affected most residents in the influenza A-negative febrile group, because the direct enzyme immunoassay of throat swab specimens showed some false negative results. But we could not rule out influenza B infection in the population completely. In this outbreak of influenza A, we could initiate oseltamivir therapy for most cases within 24 hours from symptom onset. Initiation at 0 hours, 112 hours, 13-24 hours or 72 hours from onset of symptoms was associated with mean fever durations of 26 hours, 38 hours, 54 hours and 120 hours, respectively. Earlier initiation of therapy was associated with faster resolution of fever in elderly patients. The result is in accordance with the results of healthy adults with naturally acquired febrile influenza (16, 18). In addition, no influenza symptom except low-grade fever in 2 cases developed in the influenza A-positive afebrile group (10 cases), when oseltamivir was initiated at $75 \mathrm{mg}$ twice daily for 5 days, suggesting that oseltamivir administration almost completely inhibits symptom onset of influenza. In addition, oseltamivir administration may significantly reduce the risk of illness in elderly persons who had household contacts of infected persons because none of 26 afebrile elderly residents (influenza A-negative) showed influenza-like symptoms including fever when oseltamivir $75 \mathrm{mg}$ was administered twice daily for 5 days. This study also demonstrated that oseltamivir 75 $\mathrm{mg}$ twice daily for 5 days was well tolerated in elderly patients, when oseltamivir was taken with food. In the elderly persons, adverse events of oseltamivir seem to be milder than in young adults; only 2 persons complained of nausea in 263 elderly persons (10).

We concluded that oseltamivir $75 \mathrm{mg}$ twice daily for 5 days was effective for the reduction of influenza $A$ infection or for household prophylaxis of influenza A infection in elderly persons and was well tolerated. Earlier initiation of oseltamivir therapy was associated with faster resolution in the elderly persons.

\section{References}

1) Han SN, Meydani SN. Antioxidants, cytokines, and influenza infection in aged mice and elderly humans. J Infect Dis 182 Suppl 1: S74-80, 2000.

2) Centers for Disease Control and Prevention. Prevention and control of influenza: recommendations of the advisory committee on immunization practices (ACIP). MMWR Morb Mortal Wkly Rep 47: 1-26, 1998.

3) Nichol KL, Wuorenma J, von Sternberg T. Benefits of influenza vaccination for low-, intermediate-, and high-risk senior citizens. Arch Intern Med 158: 1769-1776, 1998.

4) Nicholson KG. Use of antivirals in influenza in the elderly: prophylaxis and therapy. Gerontology. 42: 280-289, 1996.

5) Colman PM. Neuramidase: Enzyme and Antigen. in: The Influenza Viruses, Krug RM, Ed. Plenum Press, New York, 1989: 175-218.

6) von Itzstein M, Wu WY, Kok GB, et al. Rational design of potent sialidasebased inhibitors of influenza virus replication. Nature 363: 418-423, 1993.

7) Kim CU, Lew W, Williams MA, et al. Influenza neuraminidase inhibitors possessing a novel hydrophobic interaction in the enzyme active site: design, syshesis, and structural analysis of carbocylic sialic acid analogues with potent anti-influenza activity. J Am Chem Soc 19: 681-690, 1997.

8) The MIST (Management of Influenza in the Southern Hemisphere 


\section{ShiJubo et al}

Trialists) Study Group. Randomised trial of efficacy and safety of inhaled zanamivir in treatment of influenza A and B virus infections. Lancet 352: 1877-1881, 1999.

9) Li W, Escarpe PA, Eisenberg EJ, et al. Identification of GS4104 as an orally bioavailable prodrug of the influenza virus neuraminidase inhibitor GS4071. Antimicrob Agents Chemother 42: 647-653, 1998.

10) Parker R, Loewen N, Skowronski D. Experience with oseltamivir in the control of a nursing home influenza B outbreak. Can Commun Dis Rep 27: 37-40, 2001.

11) Outbreak of influenza A in a nursing home-New York, December 1991January 1992. Can Commun Dis Rep 18: 78-80, 1992.

12) Kashiwagi S, Ikematsu H, Hayashi J, Nomura H, Kajiyama W, Kaji M. An outbreak of infuenza A (H3N2) in a hospital for the elderly with emphasis on pulmonary complications. Jpn J Med 27: 177-182, 1988.

13) Tada T. Nutrition and the immune system in aging: an overview. Nutr Rev 50: 360, 1992.

14) Miller RA. The aging immune system: primer and prospectus. Science 273: 70-74, 1996.

15) Hayden FG, Treanor JJ, Fritz RS, et al. Use of the oral neuraminidase inhibitor oseltamivir in experimental human influenza: randomized controlled trials for prevention and treatment. JAMA 282: 1240-1246, 1999.

16) Nicholson KG, Aoki FY, Osterhaus AD, et al, and Neuraminidase Inhibitor Flu Treatment Investigator Group. Efficacy and safety of oseltamivir in treatment of acute influenza: a randomised controlled trial. Lancet 355 : 1845-1850, 2000.

17) Treanor JJ, Hayden FG, Vrooman PS, et al, and US Oral Neuraminidase Study Group. Efficacy and safety of the oral neuraminidase inhibitor oseltamivir in treating acute influenza: a randomized controlled trial. JAMA 283: 1016-1024, 2000.

18) McClellan K, Perry CM. Oseltamivir: a review of its use in influenza. Drugs 61: 263-283, 2001.

19) Nichol KL, Margolis KL, Wuorenma J, Von Sternberg T. The efficacy and cost effectiveness of vaccination against influenza among elderly persons living in the community. N Engl J Med 331: 778-784, 1994.

20) Gross PA, Hermogenes AW, Sacks HS, Lau J, Levandowski RA. The efficacy of influenza vaccine in elderly persons. A meta-analysis and review of the literature. Ann Intern Med 123: 518-527, 1995. 